\title{
Multi-Hop Routing Cooperative Communication for Improving Throughput in IEEE 802.11x Wireless LAN
}

\author{
Chang-Bin Ha, Hyoung-Kyu Song* \\ UT Communication Research Institute, Sejong University, Seoul, Korea (E-mail: zhkdldj@naver.com) \\ UT Communication Research Institute, Sejong University, Seoul, Korea (E-mail: songhk@sejong.ac.kr)
}

\begin{abstract}
In this paper, we propose cooperative communication scheme using multiple relays to improve the throughput of IEEE $802.11 \mathrm{x}$ wireless LAN. The proposed scheme performs cooperative communication with a terminal supporting a high data rate in order to solve the problem that a total network throughput is greatly reduced when a terminal supporting a low data rate occupies a channel. Also, the multi-hop routing is performed to optimize the performance of cooperative communication. According to the simulation results, the proposed cooperative communication scheme improves the reduction rate of the throughput even if the number of terminals supporting a low data rate increases in the entire network.
\end{abstract}

Keywords - IEEE $802.11 \mathrm{x}$, cooperative communication, throughput, multi-hop routing

\section{INTRODUCTION}

IEEE $802.11 x$ wireless local area network (LAN) has been built as a broad network infrastructure based on access points (APs) and continuously expanding. Although IEEE 802.11, which was released as the first protocol of IEEE $802.11 x$ in 1997 , supported only two data rates of 1 Mbps and $2 \mathrm{Mbps}$ in the $2.4 \mathrm{GHz}$ band, the subsequently released protocols such as IEEE 802.11a, g, n, and ac have been changed to support different data rates in according to channel condition [1]. Therefore, IEEE $802.11 \mathrm{x}$ has an advantage that can support an adaptive data rate according to the channel environment such as the number of terminals, coverage, etc. of the area where the AP is installed.

IEEE 802.11x wireless LAN ensures fair transmission of terminals by applying the same distributed coordination function (DCF) to all distributed terminals within AP coverage. When a terminal supporting a low data rate in outside of AP coverage occupies a channel, even if the data with same size is transmitted, the channel is occupied for a long time compared with a terminal supporting a high data rate [2]. Therefore, the throughput of entire network is decreased by the reduction of the available channel time for the terminals supporting a high data rate. In order to solve the problem for reduction of the total network throughput, the papers proposed the methods for setting with the different back-off slot or different frame size for each transmission group. However, these approaches reduce the transmission opportunity of terminals supporting a low data rate and it has a problem of fairness [3]. In order to solve the problem for fairness, when a relay terminal supporting a high data rate occupies a channel, the cooperative communication system that transmits data of terminal supporting a low data rate was proposed [4].

In this paper, we propose a multi-hop routing cooperative communication scheme to reduce the decrease of the total network throughput by the terminals supporting a low data rate in the IEEE 802.11x wireless LAN.

\section{SYSTEM MODEL}

Fig. 1 shows the system model of proposed cooperative communication scheme. Within the coverage of AP, because the terminals near the AP have a less path loss in according to the distance and are less affected by the fast fading in according to path change between the AP and the terminal, it can support the modulation and coding scheme (MCS) with high data rate. However, because the terminals located outside of AP coverage have a large path loss and are vulnerable to fast fading, it can support the MCS with low data rate. 


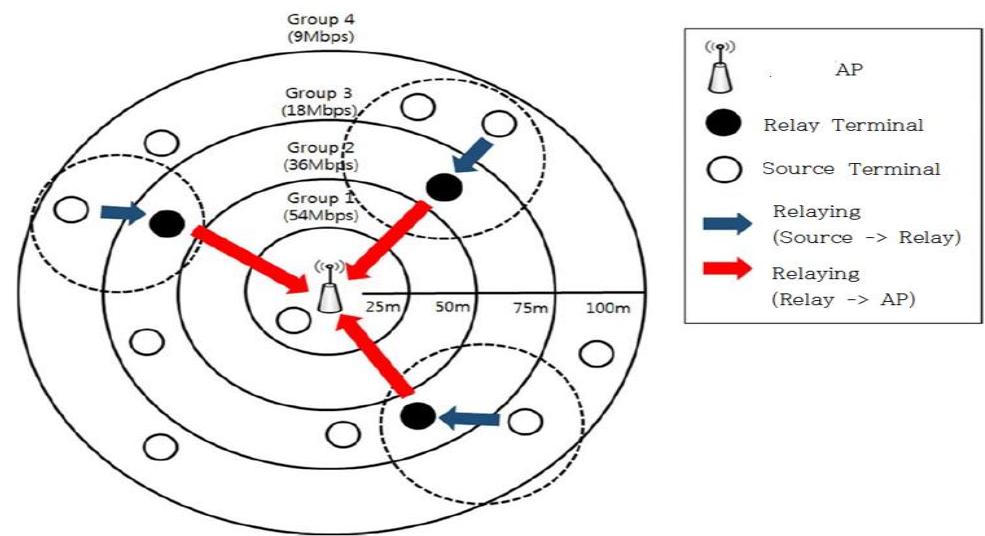

Fig. 1: Grouping model in according to AP-terminal distance in IEEE 802.11a wireless LAN

It is assumed that the protocol of the system is IEEE 802.11a. The coverage of AP is assumed to be $100 \mathrm{~m}$. Also, as shown in Fig 1, each group using MCS that supports 9Mbps, 18Mbps, 36Mbps, $54 \mathrm{Mbps}$ in according to the distance between the AP and the terminal is defined. The relay terminal is defined as a terminal belonging to group $1,2,3$, and the relay terminal transmits the data of the source terminal together with its own data.

\section{MULTI-HOP ROUTING COOPERATIVE COMMUNICATION}

The received signal of relay terminal is represented as follows,

$$
Y=H X+I+n, \quad(1)
$$

where $H$ is the impulse channel response and $X$ is the transmitted signal. Also, $I$ is the interference and $n$ is additive white Gaussian noise (AWGN). In this equation, because $I$ has a Gaussian distribution as the number of relays increases, the sum of $I$ and $n$ can be represented as Gaussian. Therefore, Eq. (1) can be represented as follows,

$$
Y=H X+N \text {, }
$$

where $N$ is a variable of Gaussian distribution for the sum of interference and AWGN.

The detection scheme is minimum mean square error (MMSE) that detects signal using $N$. The purpose of MMSE detection is to minimize mean square error (MSE) of the received signal and transmittable signal, together with Gaussian distribution. It can be represented as follows,

$$
G=\operatorname{inv}\left(H^{H} H+\sigma^{2} I\right) H^{H}
$$

where $\sigma^{2}$ is the variance of noise, $I$ is a unit matrix, and ()$^{H}$ is Hermitian operation. By multiplying the received signal with $G$ and performing hard decision in according to the modulation scheme, the demodulation is possible.

Fig. 2 shows the routing process in according to the time slot. The source terminal in the group 4 transmits the signal to the relay terminals in the group 1, 2, and 3 during the 1st time slot. The relay terminals that receive signal from the source terminal during the 1 st time slot transmit the channel state between the source terminal and itself to the relay terminals in the group 1 and 2 during the 2nd time slot. Also, the relay terminals that already receive the signal from the source terminal ignore the signal from the relay terminals. Sequentially, during the 3rd time slot, the relay terminals that receive signal during the 2 nd time slot transmit the channel state for the passed route during the 1st and the 2 nd time slot to the relay terminals in group 1 and 2 . 


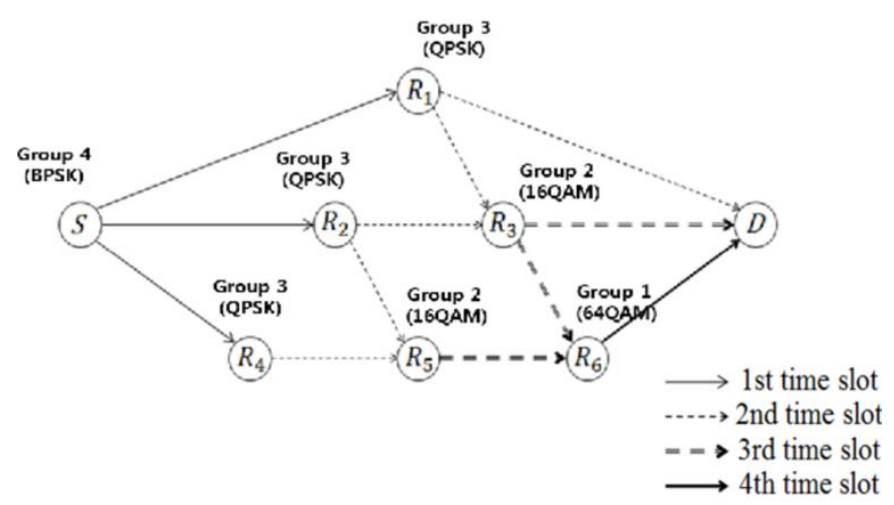

Fig. 2: Route search process in according to time slot

Finally, during the 4 th time slot, the relay terminals that receive signal during the 3 rd time slot transmit the channel state for the passed route during the $1 \mathrm{st}$, the $2 \mathrm{nd}$, and $3 \mathrm{rd}$ time slot to the relay terminals in group 1.

The best way to select a route is to compare the magnitude of the channel for each route. The AP acquires the average of the channel magnitude for each route in according to the number of relay terminals and selects the route with the largest value. This can be represented as follows,

$$
\begin{aligned}
H_{\text {sel }}= & \max \left(\alpha H_{1}, \beta H_{2}, \gamma H_{3}, \varepsilon H_{4}\right), \text { (4) } \\
& \text { where } H_{\text {sel }} \text { is the average of the channel }
\end{aligned}
$$
magnitude of selected route and $\mathrm{H}_{1}, \mathrm{H}_{2}, \mathrm{H}_{3}$, $\mathrm{H}_{4}$ are the average of acquired channel magnitude during the 1 st, the 2 nd, the $3 \mathrm{rd}$, and the 4 th time slot, respectively. Also, $\alpha, \beta, \gamma, \varepsilon$ are the value that adjusts the average of channel magnitude in according to the number of relay terminals. Because there is a possibility of demodulation error in each relay terminal, the weight is given to the route with fewer relay terminals.

\section{SIMULATION RESULTS}

Because the throughput of the entire network is significantly reduced by the terminals supporting a low data rate, the simulation of the proposed cooperative communication is performed while the number of terminals in the group 1,2, and 3 is kept and the number of terminals in the group 4 is changed. The schemes compared with the proposed scheme are the conventional IEEE 802.11a and $2 \sim 4$ hop cooperative communication without the routing.

If the number of terminals in the group 4 is large in the network, the average throughput is decreased by the reduction of the available channel time for the terminals supporting a high data rate. The average throughput is improved by using cooperative communication that a terminal supporting a high data rate transmits the data of the terminal supporting a low data rate. However, if the cooperative communication is used without routing, the possibility of demodulation error in the relay terminals is increased in according to the number of terminals in the group 4. Also, the problem causes a significant performance degradation as the number of relay terminals increases. To solve the problem, the proposed scheme uses the routing scheme considering the channel of entire route and imporves the routing performance by using the weight in according to the number of relay terminals. The simulation results show that the proposed scheme improves the reduction rate of the throughput even if the number of terminals supporting a low data rate increases in the entire network

\section{CONCLUSION}

The multi-hop routing cooperative communication is proposed to minimize the reduction of the average throughput by channel occupation of the terminal supporting a low data rate in IEEE $802.11 \mathrm{x}$ wireless LAN. The proposed scheme allows terminals supporting a high data rate to transmit data of a terminal supporting a low data rate. The route of cooperative communication is determined by routing considering the entire channel of transmitted data. Also, the weighting is used to improve the possibility of demodulation error at each relay terminal. According to the simulation results, the proposed cooperative communication scheme improves the reduction rate of the throughput even if the number of terminals supporting a low data rate increases in the entire network. 


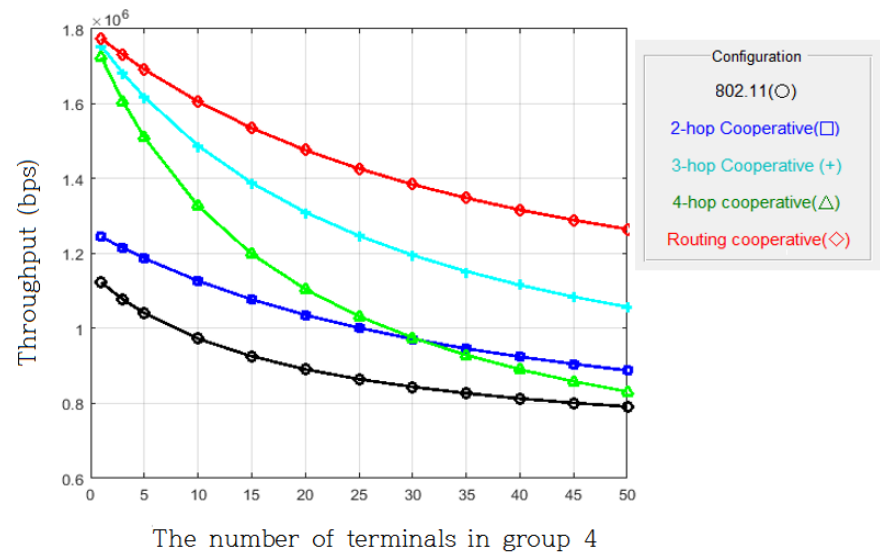

Fig 3: Comparison of proposed and conventional scheme

\section{ACKNOWLEDGEMENTS}

This work was supported by the Korea Institute of Energy Technology Evaluation and Planning(KETEP) and the Ministry of Trade, Industry \& Energy(MOTIE) of the Republic of Korea (No. 20161210200670) and was supported by the IT R\&D program of MOTIE/KEIT [10054819, Development of modular wearable platform technology for the disaster and industrial site]. (Corresponding Author: Hyoung-Kyu Song)

\section{REFERENCES}

[1] IEEE Std 802.11a-1999(R2003). Supplement to IEEE Standard for Information TechnologyTelecommunications and Information Exchange between Systems- Local and Metropolitan Area Networks-Specific Requirements. Part 11: Wireless LAN
Medium Access Control (MAC) and Physical Layer (PHY) Specifications: HighSpeed Physical Layer in the $5 \mathrm{GHz}$ Band.

[2] G. Bianchi, Performance Analysis of the IEEE 802.11 Distributed Coordination Function, IEEE Journal on Selected Areas in Communications, 18(3), 2000, 535-547.

[3] D. Y. Yang, T. J. Lee, J. B. Chang, and S. H. Choi, Performance Enhancement of Multirate IEEE 802.11 WLANs with Geographically Scattered Stations. IEEE Transcations on Mobile Computing, 5(7), 2006, 906-919.

[4] J. S. Kim, T. J. Lee, Performance Enhancement of IEEE 802.11b WLANs Using Cooperative MAC Protocol, Computational Science and Its Applications-ICCSA, 2009, 335-344. 